\title{
Antimicrobial susceptibility of gram-positive and gram-negative bacteria: a 5-year retrospective analysis at a multi-hospital healthcare system in Saudi Arabia
}

\author{
Saad Alhumaid ${ }^{1 *}$ (D), Abbas Al Mutair ${ }^{2,3}$, Z Zainab Al Alawi ${ }^{4}$, Ahmad J. Alzahrani ${ }^{5}$, Mansour Tobaiqy ${ }^{6}$, \\ Ahmed M. Alresasi ${ }^{1}$, Ibrahim Bu-Shehab ${ }^{1}$, Issa Al-Hadary', Naif Alhmeed ${ }^{7}$, Mossa Alismail ${ }^{8}$, Ahmed H. Aldera ${ }^{9}$, \\ Fadhil AlHbabi ${ }^{10}$, Haifa Al-Shammari ${ }^{11}$, Ali A. Rabaan ${ }^{12}$ and Awad Al-Omari ${ }^{13,14}$
}

\begin{abstract}
Background: Studying time-related changes in susceptible pathogens causing healthcare-associated infections (HAls) is vital in improving local antimicrobial and infection control practices.

Objectives: Describe susceptibility patterns to several antimicrobials in gram-positive and gram-negative pathogens isolated from patients causing HAls at three private tertiary care hospitals in Saudi Arabia over a 5-year period.

Methods: Data on trends of antimicrobial susceptibility among bacteria causing HAls events in children and adults at three tertiary private hospitals located in Riyadh and Qassim, Saudi Arabia, were collected retrospectively between 2015 and 2019 using the surveillance data datasets.

Results: Over a 5-year period, 38,624 pathogens caused 17,539 HAl events in 17,566 patients. About 9450 (53.8\%) of patients who suffered HAls were females and the average age was $41.7 \pm 14.3$ years (78.1\% were adults and $21.9 \%$ were children). Gram-negative pathogens were 2.3-times more likely to cause HAls compared to gram-positive bacteria (71.9\% vs. 28.1\%). The ranking of causative pathogens in decreasing order was: Escherichia coli (38\%), Klebsiella species (15.1\%), and Staphylococcus aureus (12.6\%). Gram-positive isolates were mostly susceptible to linezolid (91.8\%) whereas they were resistant to ampicillin (52.6\%), cefoxitin (54.2\%), and doxycycline (55.9\%). Gram-negative isolates were mostly sensitive to tigecycline (95\%) whereas they were resistant to cefotaxime (49.5\%) and cefixime (59.6\%). During the 5 years, there were relatively stable susceptibility patterns to all tested antimicrobials, except for cefotaxime which shown a susceptibility reduction by $41.4 \%$, among Escherichia coli and Klebsiella species. An increase in the susceptibility of Acinetobacter and Enterobacter and Citrobacter species to all studied antimicrobials was observed except for colistin that had a slight sensitivity reduction in 2019 by $4.3 \%$ against Acinetobacter species. However, we noted reduced sensitivity of MRSA, CoNS and Enterococcus species to gentamicin; and increased resistance of MRSA to linezolid and vancomycin.
\end{abstract}

Conclusion: The observed increase in susceptibility of gram-positive and gram-negative bacteria to studied antimicrobials is important; however, reduced sensitivity of MRSA, CoNS and Enterococcus species to gentamicin; and

*Correspondence: saalhumaid@moh.gov.sa

${ }^{1}$ Administration of Pharmaceutical Care, Alahsa Health Cluster, Ministry of Health, Rashdiah Street, P. O. Box 12944, Alahsa 31982, Saudi Arabia

Full list of author information is available at the end of the article

c) The Author(s) 2021. This article is licensed under a Creative Commons Attribution 4.0 International License, which permits use, sharing, adaptation, distribution and reproduction in any medium or format, as long as you give appropriate credit to the original author(s) and the source, provide a link to the Creative Commons licence, and indicate if changes were made. The images or other third party material in this article are included in the article's Creative Commons licence, unless indicated otherwise in a credit line to the material. If material is not included in the article's Creative Commons licence and your intended use is not permitted by statutory regulation or exceeds the permitted use, you will need to obtain permission directly from the copyright holder. To view a copy of this licence, visit http://creativecommons.org/licenses/by/4.0/. The Creative Commons Public Domain Dedication waiver (http://creativecommons.org/publicdomain/zero/1.0/) applies to the data made available in this article, unless otherwise stated in a credit line to the data. 
increased resistance of MRSA to linezolid and vancomycin is a serious threat and calls for effective antimicrobial stewardship programs.

Keywords: Antibiotics, Antimicrobials, Gram-positive, Gram-negative, Healthcare-associated infections, Rates, Saudi Arabia, Sensitivity, Susceptibility

\section{Background}

Antimicrobial resistance (AMR) is a major threat to public health imposing significant health and economic burdens on healthcare system and patients $[1,2]$. Unless proactive solutions are found to address AMR, global costs are estimated to reach USD 3 trillion annually by 2050 and an additional 10 million people could die each year; cumulated costs could reach over USD 100 trillion [3]. Decreasing private sector investment in the development of new antimicrobials to treat AMR infections threatens global efforts to fight this danger; and AMR requires international attention and collaboration, because bacteria do not recognize borders. In Saudi Arabia, misuse of antimicrobials is high and complicated primarily because antibiotics are available to buy by anyone over-the-counter via the community pharmacies without a legal prescription [4]. Only two years ago, Saudi Ministry of Health has implemented a nationwide ban on the sale of antibiotics without a legal prescription; however, despite this law, dispensing antibiotics without prescription is still common [4]. Routine clinical microbiology laboratory data provide a profile of the susceptibilities of specific bacteria to antimicrobial agents for monitoring and responding to emerging antimicrobial issues. Data can be utilized to help in the selection of empirical therapy by selecting the most appropriate antibiotics before susceptibility results are available, but remains generally unexploited for purposes of epidemiological surveillance. Although Antimicrobial stewardship programs focus on antibiotic prescribing practice, it is supported by an understanding of local antibiotic susceptibility trends, which in turn depends on the availability of a reliable medical microbiology laboratory resource. The Medical Group has implemented antimicrobial stewardship (AMS) programs since January 2014 and employs various strategies to reduce inappropriate utilization of antimicrobials, minimize the emergence of AMR and lower incidence of health-care-associated infections (HAIs) and reduce cost $[1,5]$.

Several local studies have estimated the rates of susceptibility among gram-positive and gram-negative bacteria in Saudi Arabia [6-10], but none was comprehensive, and comparisons are complicated by variable methods and study periods that influence the findings explanation and interpretation.

\section{Aim}

This study aimed to examine patterns of antimicrobial susceptibility of gram-positive and gram-negative pathogens isolated from inpatients and outpatients causing HAIs using the surveillance data datasets collected from three HMG hospitals (Altakhassusi, Arryan and Qassim) over a 5-year period, in Saudi Arabia.

\section{Settings}

The private tertiary medical group is considered as one of the largest private healthcare providers in the Middle Eastern region. Currently, the medical group operates 14 medical facilities across Saudi Arabia, UAE and Bahrain, including 7 hospitals and 6 medical centers.

Study was conducted at three tertiary and specialized health facilities with adequate medical professional resources with 237-bed capacity, 365-bed and 150-bed capacity, respectively located in two different cities in Saudi Arabia.

These facilities provide healthcare services to a wide range of patients in various specialties and subspecialties. Yearly, the three healthcare facilities encounter over 127,364 surgical cases, nearly $1,742,144$ visits to emergency departments, and over 360,587 admissions.

\section{Methods \\ Study design}

Data of trends in antimicrobial susceptibility among of all reports of four types of gram-positive isolates [Staphylococcus aureus, Methicillin-resistant Staphylococcus aureus (MRSA), Coagulase-negative staphylococci (CoNS) and Enterococcus species] and six types of gram-negative isolates [Escherichia coli, Klebsiella species, Pseudomonas species, Acinetobacter species, Proteus species, and Enterobacter and Citrobacter species] causing HAIs, collected from the infection control and prevention surveillance data between January 2015 and December 2019 from adult and pediatric patients in three tertiary private hospitals in Saudi Arabia, were extracted using standard customized Excel data collection sheets (Microsoft Corp, Redmond, WA, USA). The antimicrobial susceptibility patterns for selected antimicrobials were analyzed and reported.

We extracted the following patient data from the patient records meeting the inclusion criteria: age, gender, patient location (wards, intensive care units, and 
outpatient settings), specimen type, HAI type, organism identified, and antimicrobial susceptibility test results.

\section{Inclusion-exclusion criteria}

Data on incidence of targeted bacterial isolates causing HAIs and susceptibility trends of selected pathogens to various antimicrobials collected from medical and surgical wards, intensive care units (ICUs), emergency rooms and hospital-affiliated outpatient clinics from inpatients and outpatients with blood, urinary, rectal, cerebral spinal fluid, respiratory, saliva, nasal, cervical, lavages, wound, tissue, and semen cultures (consecutive, one per patient, per infection site) were included.

Representatives from all clinically important antimicrobial classes have been tested (ampicillin, cloxacillin, amoxicillin/clavulanic acid, piperacillin/tazobactam, cefoxitin, cefazolin, cefuroxime, cefixime, cefotaxime, ceftriaxone, ceftazidime, cefepime, ciprofloxacin, levofloxacin, ofloxacin, nitrofurantoin, erythromycin, clindamycin, trimethoprim-sulfamethoxazole, amikacin, gentamicin, doxycycline, tetracycline, vancomycin, linezolid, imipenem, meropenem, tigecycline and colistin).

Infection events and response of pathogens to antibacterials lacking microorganism and/or culture and sensitivity testing information were excluded.

\section{Antimicrobial susceptibility testing}

Species identification of isolates and their antimicrobial susceptibility profiles were obtained with different automated systems at every single laboratory of the three facilities using (VITEK ${ }^{\circledR} 2$ system, BioMariex, France), BD Phoenix system (BD Biosciences, NJ, USA), MicroScan plus (Beckman Coulter, CA, USA), and BD BACTEC system (BD Biosciences) according to manufacturers' specifications, between 2015 and 2019, with susceptibility interpretations based on the Clinical and Laboratory Standards Institute (CLSI) broth microdilution and breakpoint criteria [11]. To ensure data compatibility, quality control was performed using control strains from the following American Type Culture Collection (ATCCs): Staphylococcus aureus ATCC 29213, Pseudomonas aeruginosa ATCC 2853, Escherichia coli ATCC 25922, Escherichia coli ATCC 35218, Klebsiella pneumoniae ATCC 27736 and Enterococcus faecalis ATCC 29212. Data are only included when the quality control test results were in acceptable ranges.

\section{Statistical analysis}

Variables that were continuous were presented as means and categorical variables were presented as frequencies and percentages. Susceptibility patterns of pathogens were presented over time. The difference in sensitivity trends between 2015 and 2019 was examined using the multivariate analysis of variance (MANOVA) and a two-sided P-values $<0.05$ were considered to be statistically significant. The proportion of susceptible isolates was calculated as the sum of susceptible organisms (neither intermediately susceptible nor resistant) relative to the total number of organisms tested. SPSS (Version 25.0. Armonk, NY: IBM Corp) and Microsoft Excel Professional Plus 2019 (Microsoft Corp., Redmond, WA, USA) were used for all statistical analyses.

Our study was performed in accordance with the ethical standards of the Declaration of Helsinki and its later amendments or comparable ethical standards. Ethics approval (RC20.10.95-2) was obtained by the Ethics Committee of the coordinator center (IRB Committee of Dr. Sulaiman Al Habib Medical Group, Riyadh, Saudi Arabia).

\section{Results \\ Incidence of pathogens causing HAls and patient characteristics}

A total of 41,813 pathogens were isolated over 5 years in the three of our medical group's facilities of which 38,624 pathogens caused 17,539 HAI events in 17,566 patients. These HAIs events were contracted in HMG Hospital in Altakhassusi (6016 HAI events $=34.3 \%$ ), HMG Hospital in Arryan (5893 HAI events $=33.6 \%)$ and HMG Hospital in Qassim (5630 HAI events $=32.1 \%)$. Reported HAIs varied in type: catheter-associated urinary tract infection (CAUTI) (29.4\%), central line-associated bloodstream infection (CLABSI) (27.3\%), surgical site infection (SSI) (26.1\%) and ventilator-associated events (VAE) (17.2\%). Processed samples were blood (24.7\%), urinary (19.1\%), respiratory (13.4\%), cerebral spinal fluid (8.5\%), cervical (8.2\%), saliva (5.2\%), nasal (5.1), rectal (4.9\%), lavages $(4.7 \%)$, wound $(3.9 \%)$, tissue $(1.4 \%)$, and semen $(0.9 \%)$. These HAI events were isolated in the intensive care units (37.2\%), wards (32.9\%), and outpatients (29.9\%). In our study, we excluded 6232 (16.1\%) HAI events due to the lack of data on the antimicrobial, pathogen, and/ or culture response and sensitivity testing. About 9450 (53.8\%) of patients who suffered HAIs were identified as females and had a mean age of $41.7 \pm 14.3$ years (78.1\% were adults and $21.9 \%$ were children). Of 38,624 isolates taken from clinical specimens between 2015 and 2019, 27,754 (71.9\%) were gram-negative organisms and 10,870 (28.1\%) were gram-positive organisms. The ranking of causative pathogens in decreasing order was: Escherichia coli (38\%), Klebsiella species (15.1\%), Staphylococcus aureus (12.6\%), Pseudomonas species (10.1\%), and Enterococcus species (5.9\%) (Fig. 1). 


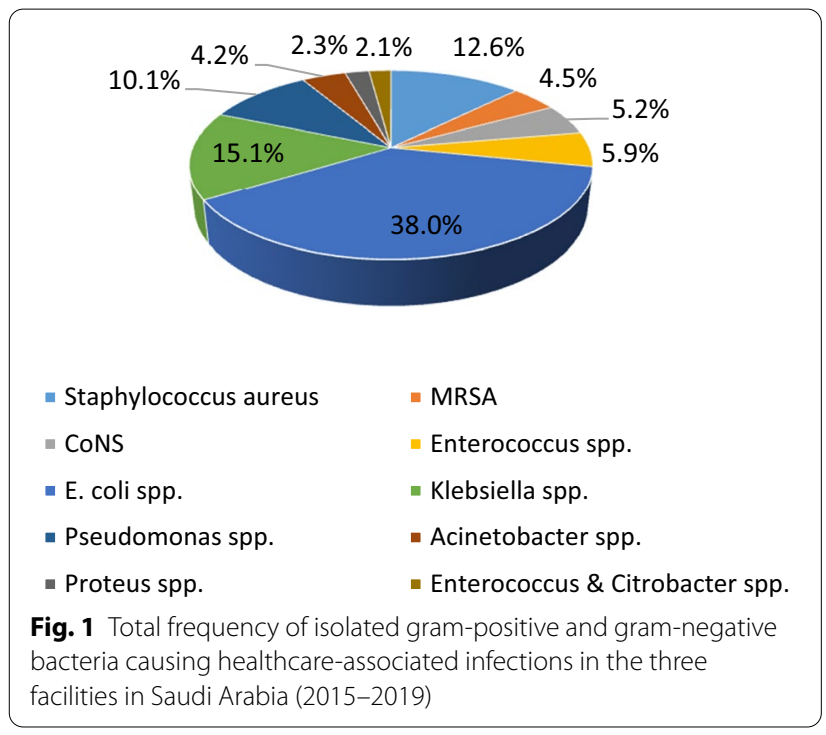

Trends of susceptibility among gram-positive bacteria

A total of 79,280 gram-positive pathogen sensitivity events against 14 clinically important antimicrobials occurred at HMG Hospital in Altakhassusi (38.2\%), HMG Hospital in Arryan (36.6\%), and HMG Hospital in Qassim (25.2\%). Gram-positive bacteria showed an overall susceptibility of $\geq 52.6 \%$. Antimicrobial susceptibility patterns in gram-positive pathogens over time are presented in Table 1.

Generally, the highest susceptibilities of gram-positive pathogens to antimicrobials were seen towards vancomycin and linezolid by Staphylococcus aureus, 98.7\% and 96.4\%; CoNS, 99.7\% and 99.6\%; and Enterococcus species, 99.5\% and 85.9\%; respectively. Moreover, Staphylococcus aureus was found to be highly sensitive to gentamicin (93.4\%), clindamycin (92\%), and erythromycin (90.5\%); MRSA was most sensitive to nitrofurantoin (82.6\%); CoNS was sensitive to doxycycline (85.1\%) and cefoxitin (81.3\%); and Enterococcus species was sensitive to ampicillin (83\%), cloxacillin (82.6\%) and nitrofurantoin $(80.9 \%)$ over the 5 -year period.

In opposite, lowest susceptibilities of gram-positive pathogens to antimicrobials were seen to ampicillin by CoNS, 15.2\%; MRSA, 19.5\%; and Staphylococcus aureus, 41.3\%; respectively. Also, Enterococcus species was least susceptible to doxycycline (17.7\%); and MRSA was slightly sensitive to cefoxitin (21.3\%).

Tetracycline, trimethoprim-sulfamethoxazole, levofloxacin and cloxacillin retained activity against $88.7 \%$, $87.7 \%, 83.1 \%$, and $82.9 \%$ of Staphylococcus aureus isolates, respectively, whereas trimethoprim-sulfamethoxazole was active against $73.6 \%$ of the CoNS isolates.
Over the 5 years, sensitivity of nitrofurantoin to overall gram-positive bacteria was the only antimicrobial to increase significantly $(30.2 \%$ increase, $\mathrm{p}$-value $=0.032)$. Prominent insignificant increase in the susceptibility of specific gram-positive bacteria to some antimicrobials occurred in 2019 compared to 2015 by: 30.5\% for Staphylococcus aureus to tetracycline; $53.1 \%$ and $45.7 \%$ for MRSA to gentamicin and clindamycin, respectively; $37.6 \%, 55.3 \%, 38.4 \%, 38.5 \%$ and $40.7 \%$ for CoNS to cefoxitin, tetracycline, clindamycin, trimethoprim-sulfamethoxazole and nitrofurantoin, respectively; $53.1 \%$, $47.9 \%$ and $48.3 \%$ for Enterococcus species to trimethoprimsulfamethoxazole, nitrofurantoin and vancomycin, respectively. However, noticeable insignificant decrease in susceptibility were seen in 2019 compared to 2015 by: 47.6\% for MRSA to cefoxitin; and 23.8\% for Enterococcus species to ciprofloxacin.

Overall, among the studied antibiotics the gram-positive isolates were mostly sensitive to linezolid (91.8\%) whereas they were resistant to ampicillin (52.6\%), cefoxitin $(54.2 \%)$, and doxycycline (55.9\%) (Table 1$)$.

\section{Trends of susceptibility among gram-negative bacteria}

A total of 314,624 gram-negative pathogen sensitivity events against 21 clinically important antimicrobials occurred at HMG Hospital in Altakhassusi (35.9\%), HMG Hospital in Arryan (39.3\%), and HMG Hospital in Qassim (24.8\%). Gram-negative bacteria showed an overall susceptibility of $\geq 49.5 \%$. Antimicrobial susceptibility patterns in gram-negative pathogens over time are presented in Table 2.

Generally, the highest susceptibilities of gram-negative pathogens to antimicrobials were seen towards: tigecycline, meropenem, imipenem and amikacin by Escherichia coli, 98.9\%, 97.5\%, 97.4\% and 96.5\%, respectively; amikacin, meropenem and colistin by Klebsiella species, 93.8\%, 92.4\% and 92.4\%, respectively; tigecycline, colistin, amikacin and gentamicin by Pseudomonas species, 98.3\%, 98.2\%, 94.9\% and 93.4\%, respectively; tigecycline and colistin by Acinetobacter species, 95.3\% and 94.6\%, respectively; colistin and tigecycline by Proteus species, 95.8\% and $95.3 \%$, respectively; and tigecycline and colistin by Enterobacter and Citrobacter sepcies,95.1\% and $94.8 \%$, respectively.

Moreover, Escherichia coli was found to be highly sensitive to nitrofurantoin (94.5\%), piperacillin-tazobactam (93.6\%), gentamicin (92.5\%) and ciprofloxacin (90.5\%); against Klebsiella species, imipenem, amoxicillin/clavulanic acid, gentamicin and piperacillintazobactam retained susceptibility $>85 \%$; Pseudomonas species were sensitive to ciprofloxacin (87.5\%), piperacillin-tazobactam (85.2\%), imipenem (83.8\%), meropenem $(82.9 \%)$ and ceftazidime (82.3\%); Proteus species 


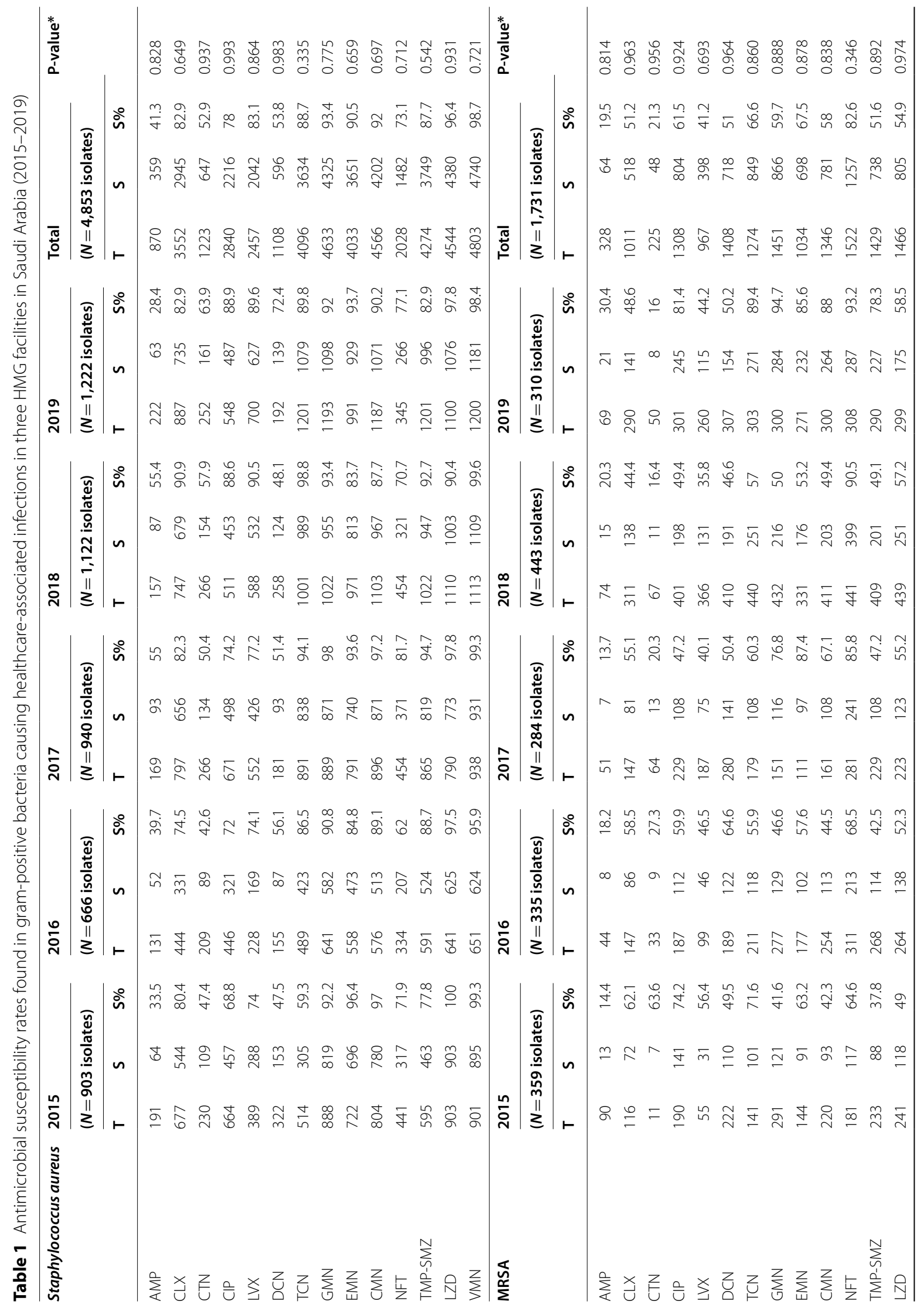




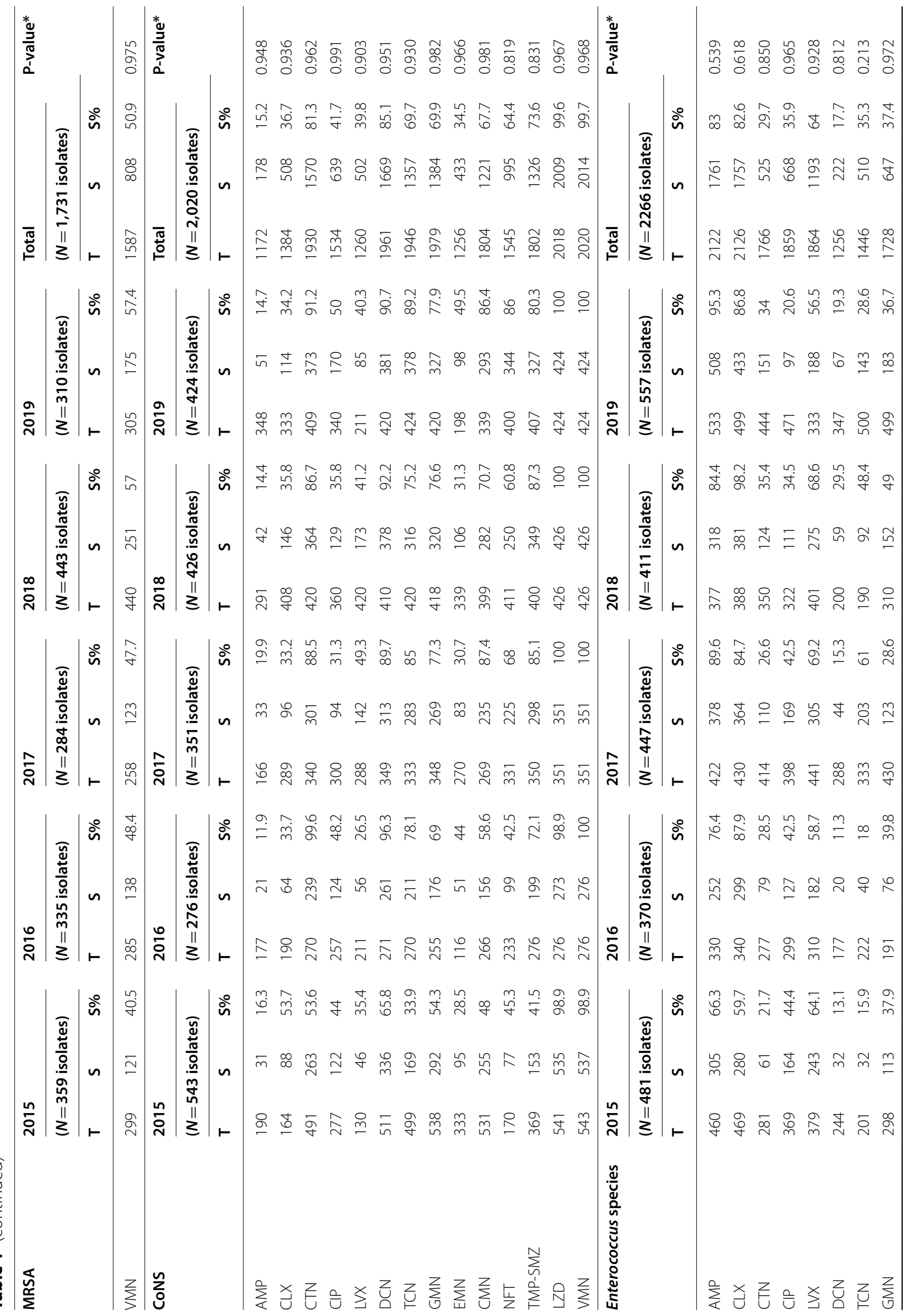




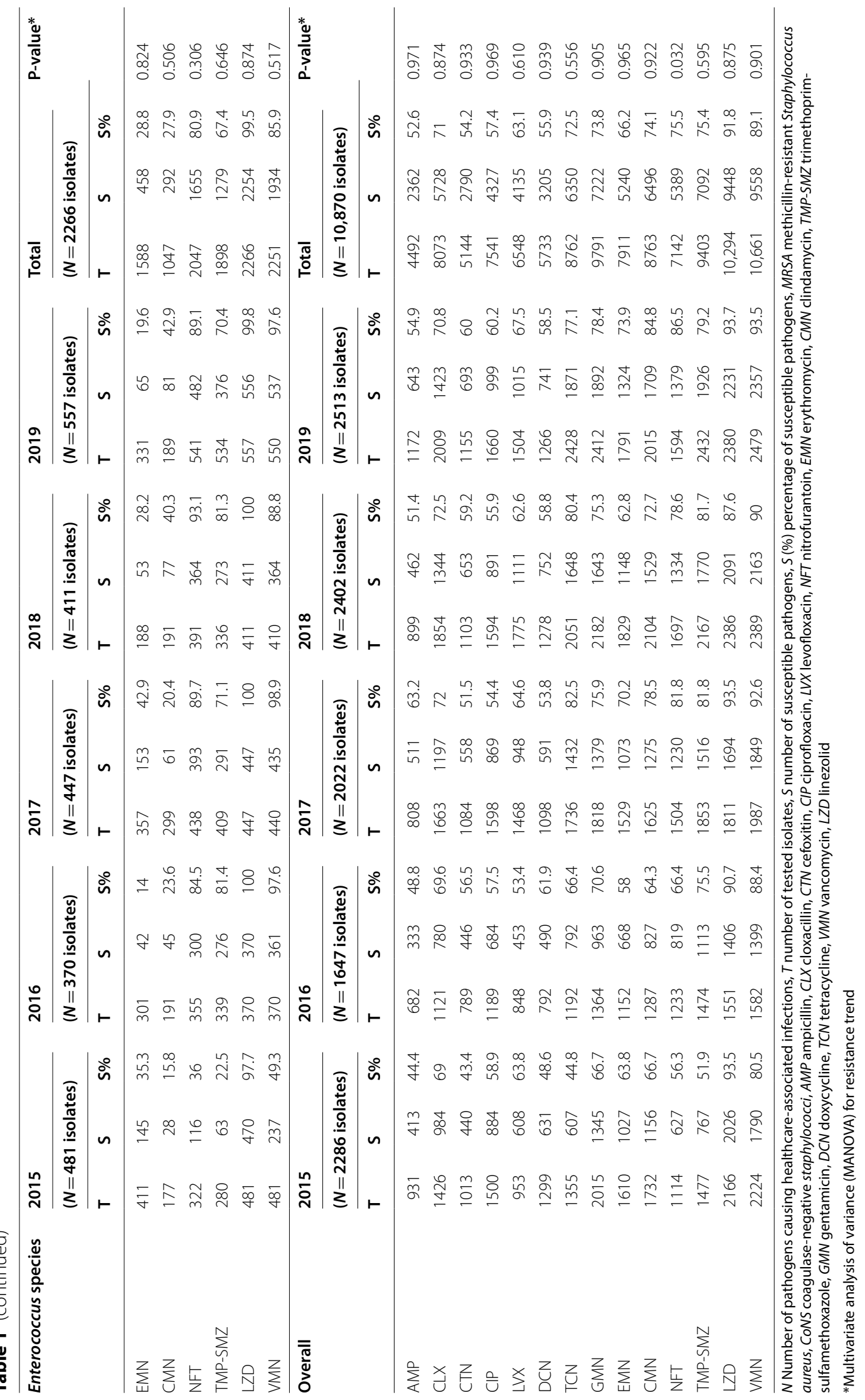




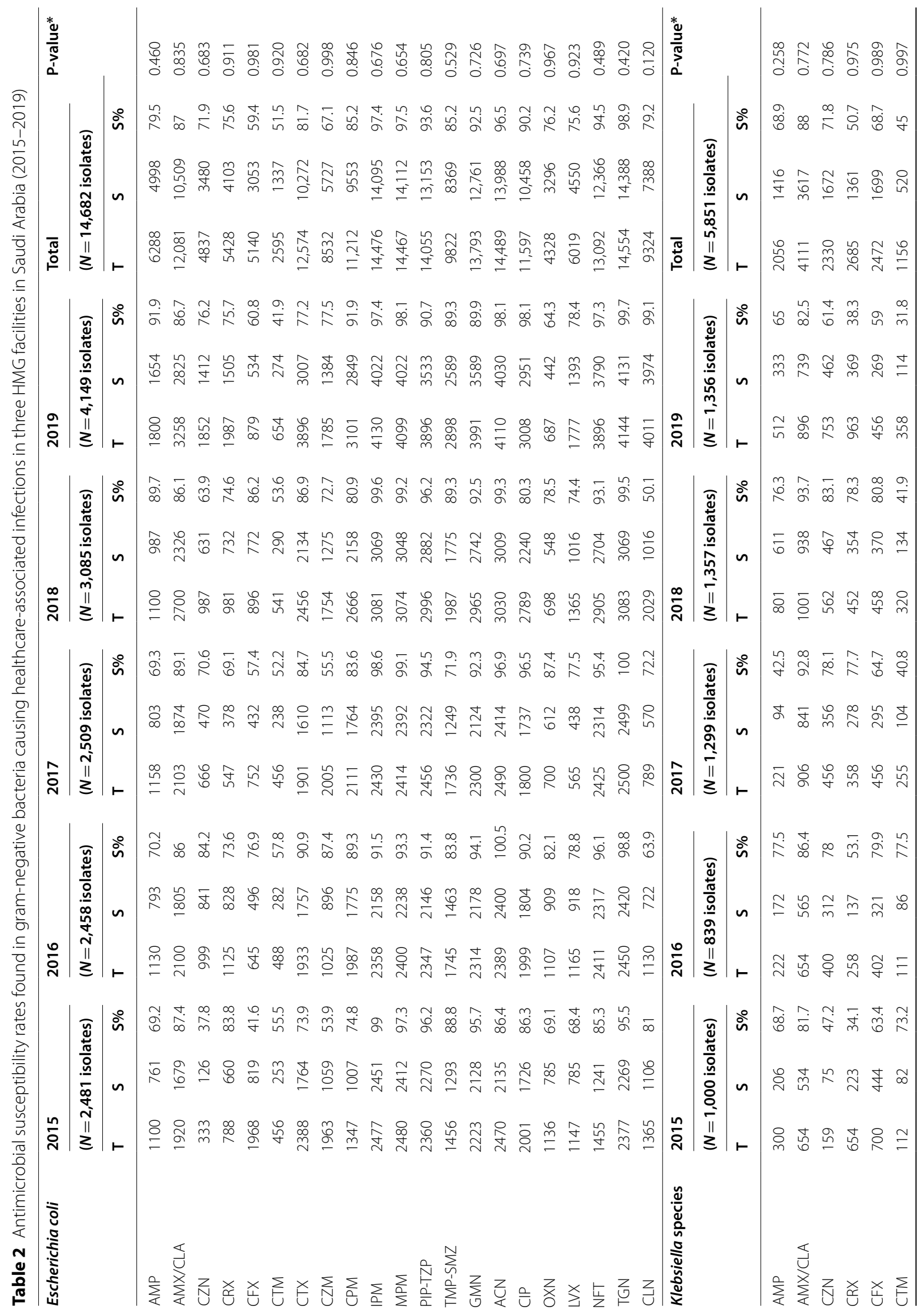




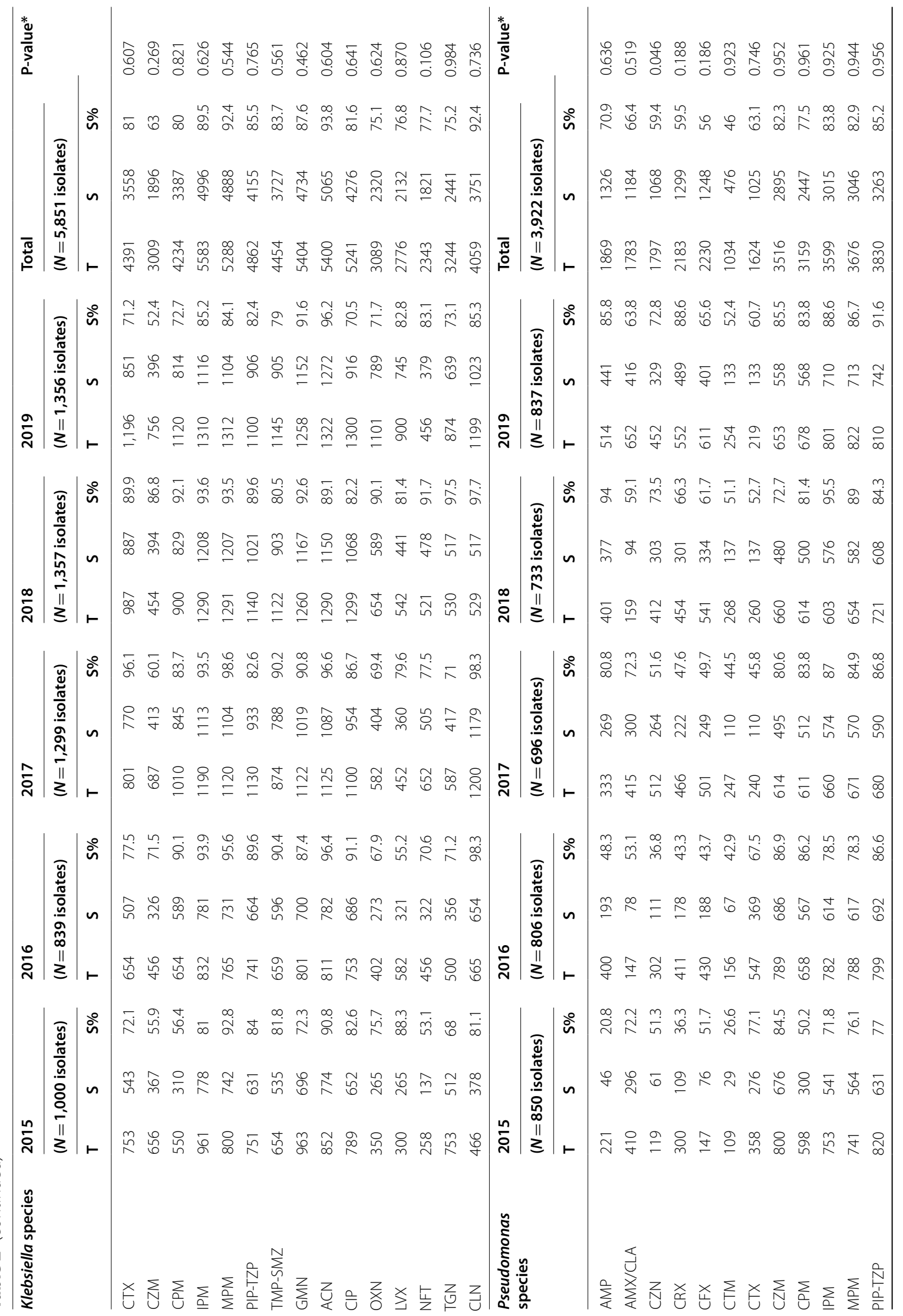




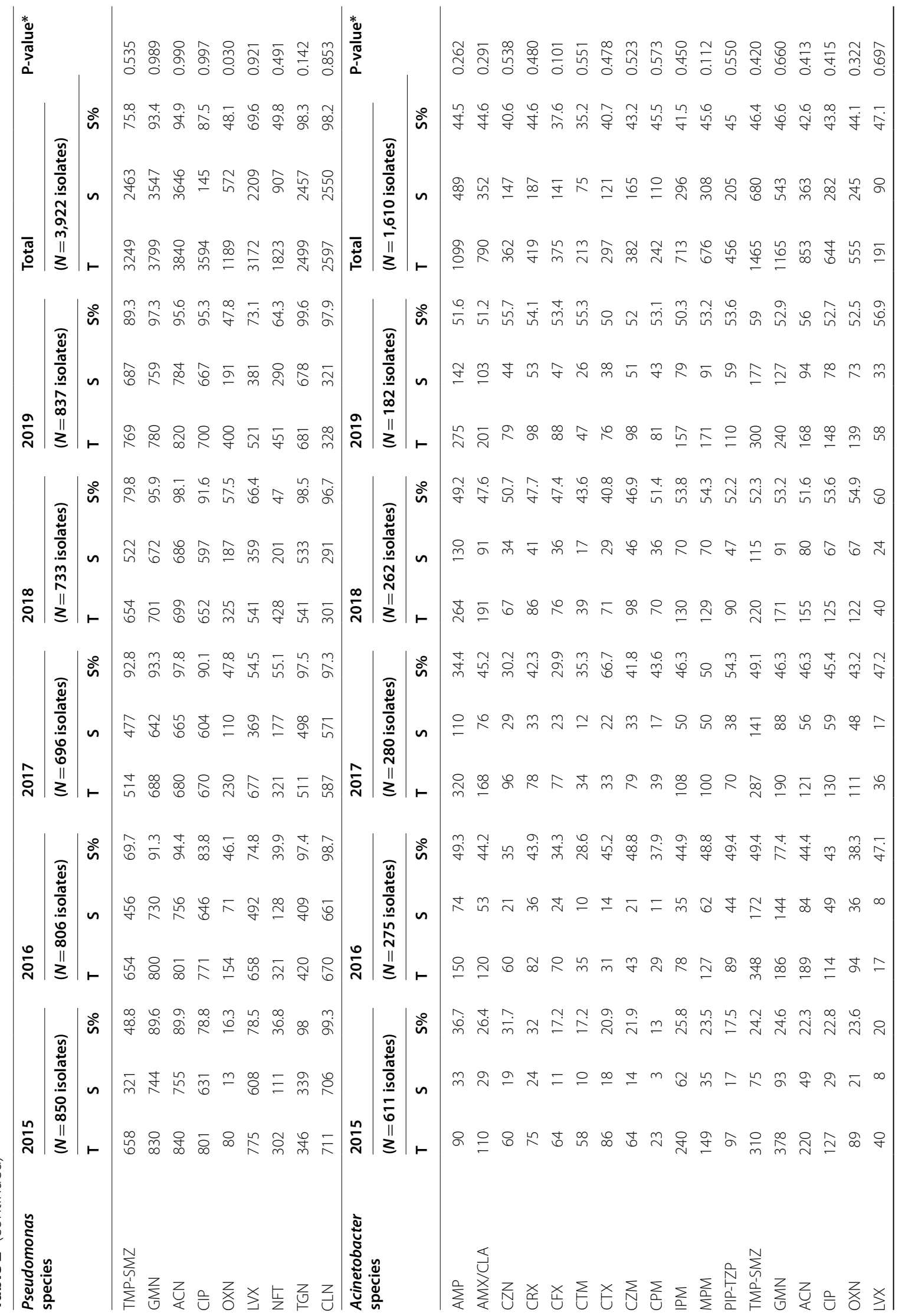




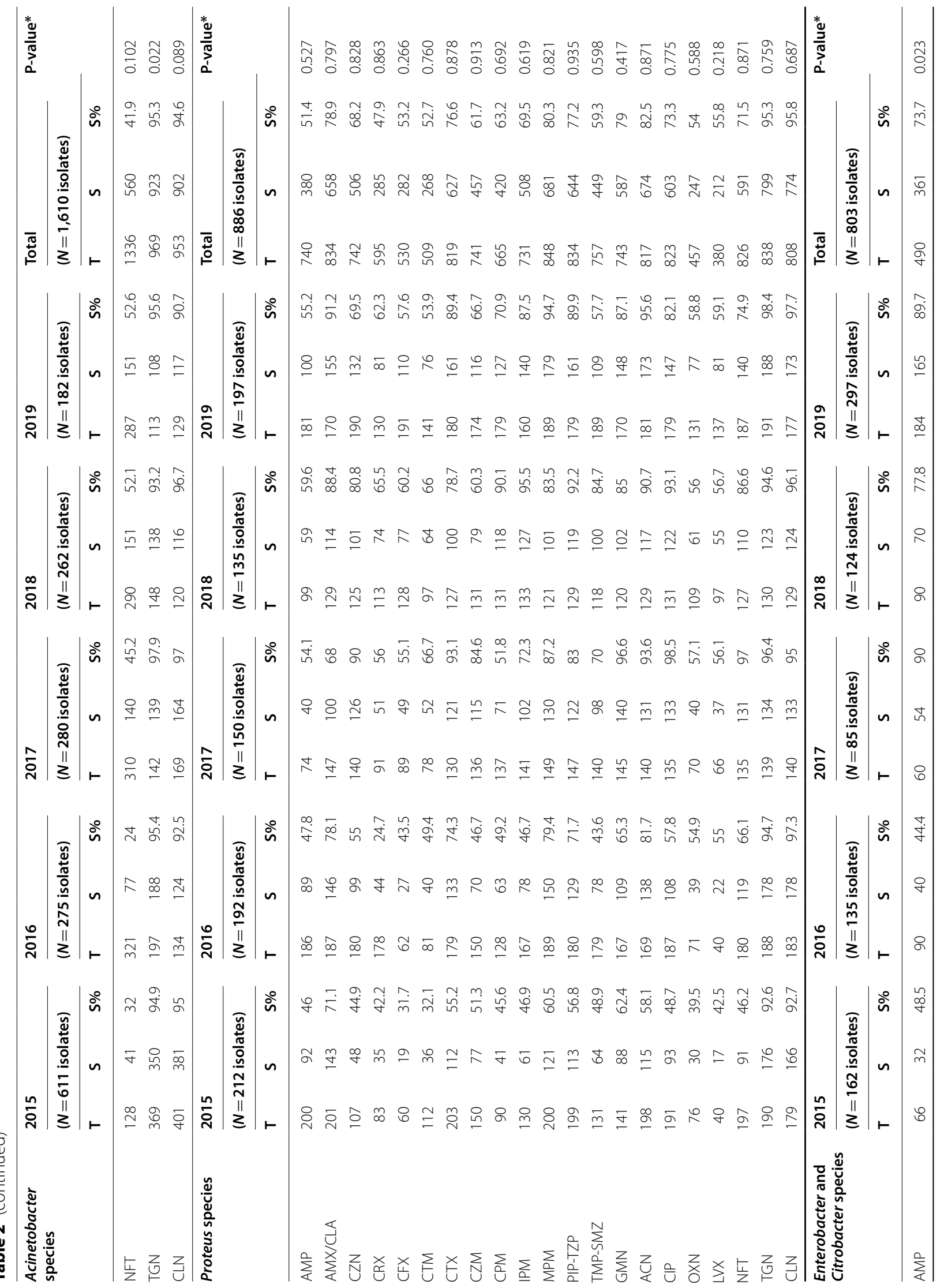




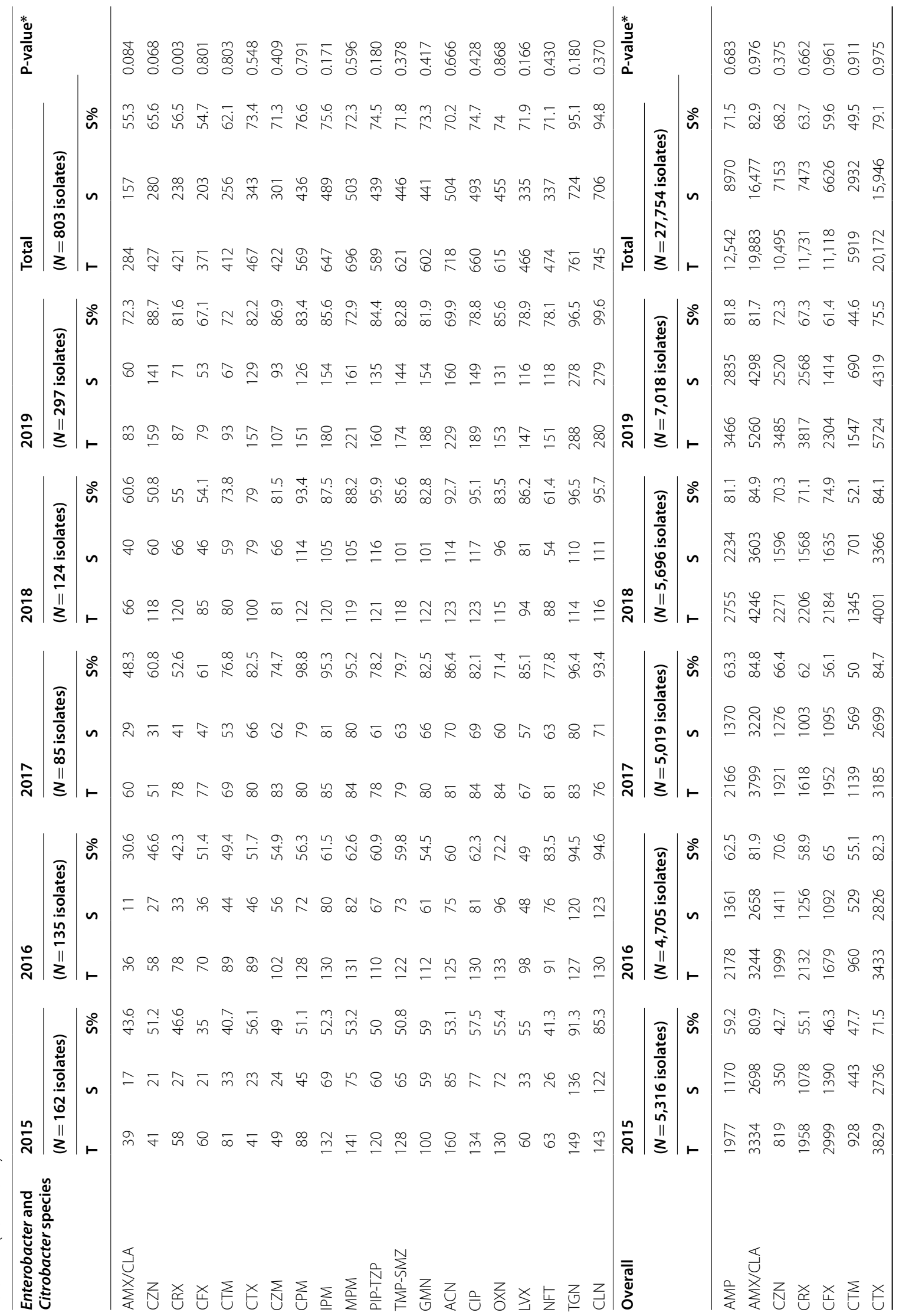




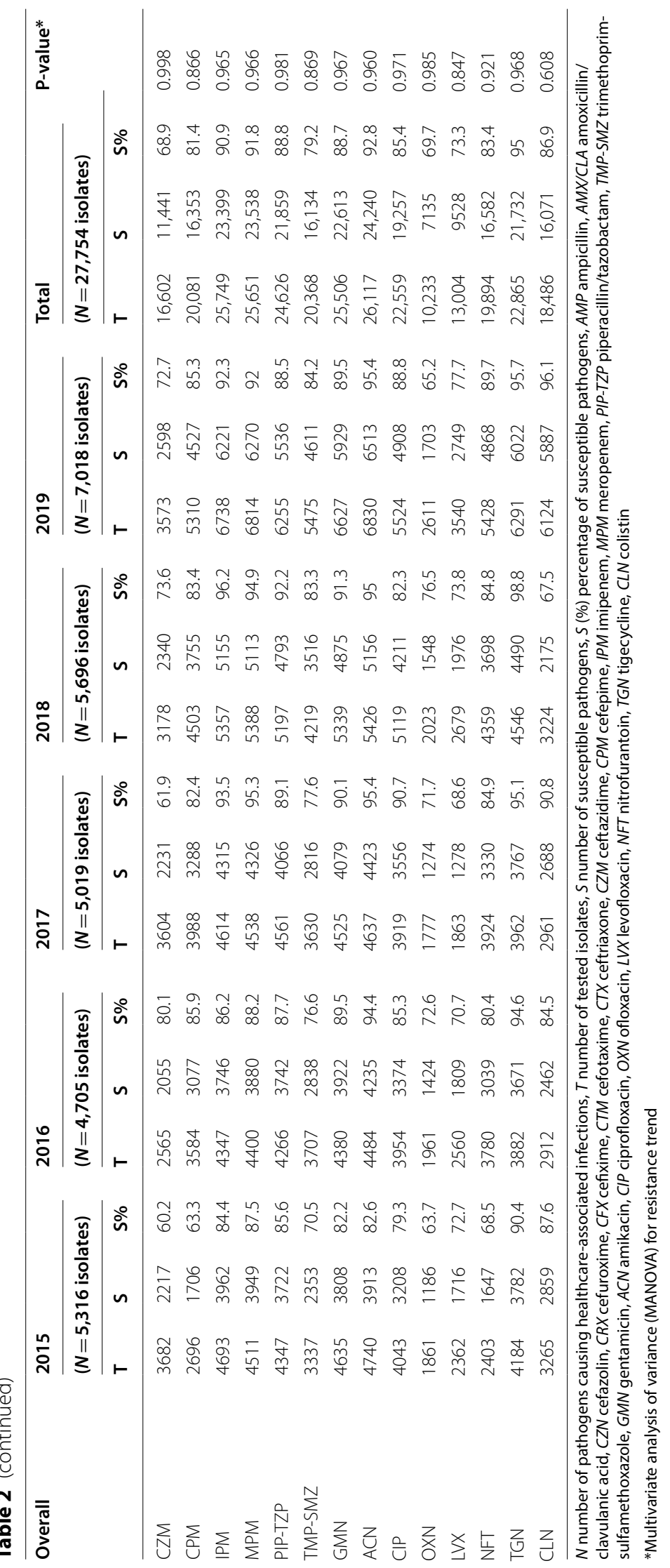


were sensitive to amikacin (82.5\%) and meropenem (80.3\%); and Enterobacter and Citrobacter species were sensitive by $\geq 70 \%$ to most of the tested antimicrobials over the 5-year period.

In contrary, lowest susceptibilities of gram-negative pathogens to antimicrobials were seen to cefotaxime and cefixime by Acinetobacter species, 35.2\% and 37.6\%, respectively. Acinetobacter species shown low sensitivity of $\geq 40 \%$ almost to all antimicrobials; and both Klebsiella and Pseudomonas species were slightly sensitive to cefotaxime ( $45 \%$ and $46 \%$, respectively).

Over the 5 years, sensitivity of cefazolin and ofloxacin to Pseudomonas species increased significantly $(21.5 \%$ and $31.5 \%$ increase, $\mathrm{p}$-values $=0.046$ and 0.030 ; respectively). The small sensitivity increase of Acinetobacter species towards tigecycline was found to be significant $(0.4 \%$ increase, $\mathrm{p}$-value $=0.022)$. In addition, large difference in susceptibility were found for both ampicillin and cefuroxime towards Enterobacter and Citrobacter species $(41.2 \%$ and $35.1 \%$ increase, $\mathrm{p}$-values $=0.023$ and 0.003 ; respectively).

Prominent insignificant increase in the susceptibility of specific gram-negative bacteria to some antimicrobials occurred in 2019 compared to 2015 by: 38.4\% for Escherichia coli to cefazolin; $30 \%$ for Klebsiella species to nitrofurantoin; and 65\%, 52.3\%, 40.6\%, 33.6\% and 31.5\% for Pseudomonas species to ampicillin, cefuroxime, trimethoprim-sulfamethoxazole, cefepime and ofloxacin, respectively.

For a 5-year difference, sensitivity of Acinetobacter species to antimicrobials shown many insignificant increases: (rate of sensitivity increase: for cefepime, 40\%; for cefotaxime, 38.1\%; for levofloxacin, 36.9\%; for cefixime, 36.2\%; for piperacillin-tazobactam, 36.1\%; for trimethoprim-sulfamethoxazole, 34.8\%; for amikacin, $33.7 \%$; and for ceftazidime, $30.2 \%$.

Enterobacter and Citrobacter species exhibited most of the sensitivity increase changes to antimicrobials of all gram-negative isolates. In 2019 compared to 2015, Enterobacter and Citrobacter species susceptibility increased insignificantly by: $41.2 \%$ for ampicillin; $37.9 \%$ for ceftazidime; $37.5 \%$ for cefazolin; $36.9 \%$ for nitrofurantoin; $35.1 \%$ for cefuroxime; $34.4 \%$ for piperacillin-tazobactam; $33.3 \%$ for imipenem; $32.3 \%$ for cefepime; $32.1 \%$ for cefixime; $32 \%$ for trimethoprim-sulfamethoxazole; $31.3 \%$ for cefotaxime; and $30.2 \%$ for ofloxacin. However, a big insignificant decrease in susceptibility was seen in 2019 compared to 2015 by cefotaxime for Klebsiella species (41.4\%).

Overall, among the studied antibiotics the gram-negative isolates were mostly sensitive to tigecycline (95\%) whereas they were resistant to cefotaxime (49.5\%) and cefixime (59.6\%) (Table 2).

\section{Discussion}

This retrospective study describes the distribution of pathogens causing HAIs and susceptibility patterns for a very high number of samples collected from both the ward and clinics in Saudi Arabia from 2015 to 2019, with an emphasis on the antibiotic classes frequently utilized to treat common infections given by a huge national surveillance program. The most commonly encountered organisms were Escherichia coli, Klebsiella species, and Staphylococcus aureus. Though various studies have previously described susceptibility rates in several infectious isolates, Saudi data are limited either to single-center studies $[7,12-20]$ or to research concentrating on the susceptibility to single or double antimicrobial classes [21-26].

One of the vital findings of the data analysis of this study was the significant increase of sensitivity for overall gram-positive bacteria to nitrofurantoin over the 5 years $(30.2 \%$ increase, $\mathrm{p}$-value $=0.032)$. Interestingly, the susceptibility of Staphylococcus aureus to tetracycline; MRSA to gentamicin and clindamycin; CoNS to cefoxitin, tetracycline, clindamycin, trimethoprim-sulfamethoxazole and nitrofurantoin; and Enterococcus species to trimethoprim-sulfamethoxazole, nitrofurantoin and vancomycin; increased insignificantly over time by $\geq 30 \%$ although this was likely due to the change of followed guidelines used for antimicrobial susceptibility testing at the Medical Group facilities, a shift from the Clinical and Laboratory Standards Institute (CLSI) to the European Committee on Antimicrobial Susceptibility Testing (EUCAST) $[11,27]$.

A comparison of the current results with findings from previous studies can offer some validation of the findings of this present study and identify methodological distinctions in their approaches. As expected, HAI events were more frequent in the ICUs (37.2\%) compared with non-ICU locations [HAI events in wards and outpatients were $32.9 \%$ and $29.9 \%$, respectively], a finding which was previously described in local studies $[10,28]$ and may reflect the epicenter role of ICU in both infections and antimicrobial resistance. The predominant isolates to cause HAIs were gram-negative organisms $(71.9 \%$ vs. $28.1 \%)$; this finding was similar with many Saudi studies made in different cities in Saudi Arabia including Riyadh [6, 28, 29], Makkah [30, 31], Dhahran [32], Bisha [33], and Aljouf [10]; with the majority being Escherichia coli (38\%) accounting approximately for $52.9 \%$ of the gram-negative bacterial growth in line with previous national studies [7, $29,30,32,34]$. The second predominant isolates of the gram-negative organisms were the Klebsiella species (15.1\%), this finding was similar to the bacterial isolates prevalence studies from Dhahran [7], and Riyadh 
[6]. The proportions of Klebsiella were $17.2 \%$ in Dhahran [7], and $14.7 \%$ in Riyadh [6]. The culture rate in our study for Proteus species (2.3\%) was comparable to previously reported rates in two different studies in Riyadh (1.2\% and 1.8\%) [6,35]. Also, the incidence of Acinetobacter species in our study was very close to the rate reported before ( $4.2 \%$ vs $5.5 \%)$ [6]; in contrast to the much higher rates found in two separate studies in Riyadh $(31.7 \%$ and $25.3 \%)$ [35, 36]. Our prevalence of Staphylococcus aureus was similar to the rate described in a previous report done in Riyadh (12.6\% vs $13.9 \%)$ [6]. We report a lower rate of MRSA (15.9\%) compared to two previous studies made in Riyadh $(24.4 \%$ and $30.3 \%$, respectively) [28] but similar to the rate reported before in another study in Riyadh (17.5\%) [29]. We report a higher susceptibility of Enterococcus species to vancomycin ( $85.9 \%$ vs $79.7 \%$ ) compared to one study in Riyadh [6]. In our study, proportion of Pseudomonas species that caused HAIs is less than what was reported in Riyadh (10.1\% vs $15.4 \%$ ) [6]; however, our prevalence was in agreement to the bacterial frequency in a study from Dhahran (12.8\%) [7]. Frequency of CoNS in causing HAIs in this study is in line with a study from Riyadh (5.2\% vs 6.5\%) [6] but much lower than the rate reported previously in a study in Riyadh (28.4\%) [29]. In our study, incidence of Enterococcus species as causative pathogens for HAIs is almost half of the reported rate by a study in Riyadh (4.5\% vs $8.6 \%$ ) [6]; however, rate was in parallel to the prevalence reported in other study in Riyadh (5.9\%) [28] but contradicts with the rate reported in another study in Riyadh (15.8\%) [29].

Our data analysis regarding the susceptibility patterns of antimicrobials confirm or contradict the findings of previous local studies. For example, Pseudomonas and Acinetobacter were most susceptible to colistin and amikacin in a study in Riyadh [29], whereas in our study, tigecycline and colistin had higher susceptibility rates. On the other hand, Escherichia coli, Klebsiella pneumonia, Enterobacter and Citrobacter species were most sensitive to amikacin, imipenem and meropenem [29], whereas in our study, Escherichia coli and Enterobacter and Citrobacter species were most susceptible to tigecycline, and Klebsiella species was most susceptible to amikacin. However, our study support the finding that CoNS were most susceptible to vancomycin and linezolid [29] and we found the susceptibility of Staphylococcus aureus to clindamycin and trimethoprim-sulfamethoxazole were almost identical to the results of the aforementioned study ( $92 \%$ vs $94 \%$ and 87.7 vs $87 \%$, respectively). This is might be due the fact that the sample was drawn from tertiary private hospitals in Saudi Arabia where the level of environmental hygiene is higher and staff are highly restricted to infection control practices.
Linezolid and vancomycin had the best susceptibility profile to Staphylococcus aureus, CoNS, and Enterococcus species while gentamicin shown low sensitivity towards MRSA, CoNS and enterococcus species. In the context of emergence of resistance of malicious grampositive bacteria to gentamicin, linezolid and vancomycin have become effective alternatives to gentamicin treatment frequently associated with nephrotoxicity [37]. Linezolid and vancomycin are active against the most serious gram-positive bacteria, including streptococci, vancomycin-resistant enterococci (VRE) and MRSA [38]. Nevertheless, we noted a low rate of susceptibility of linezolid and vancomycin against MRSA (54.9\% and 50.9\%, respectively) likely because of antibiotic selection pressure and possibly a reflection of selective reporting of susceptibility testing; this finding contradicts those of a recent study in Riyadh, which identified a $100 \%$-sensitivity of both antimicrobial agents for MRSA [29]. The relatively lower susceptibility in gram-positive bacteria in the current study may be reflecting Saudi prescription trends in recent years that overuse fluoroquinolones [1, $39]$ and carbapenems [40, 41] at the expenses of other broad-spectrum such as linezolid and vancomycin due to increased availability and reduced cost of these drugs. However, nitrofurantoin maintained the greatest efficacy against MRSA in our study (82.6\%); supporting the finding of a recent study in Aseer that shown 100\% susceptibility of MRSA to nitrofurantoin [42].

Over the 5-year period, it is interesting to note imipenem and meropenem either retained its activity or shown susceptibility increase patterns towards all the studied gram-negative pathogens except for imipenem which was less sensitive in 2019 by $1.6 \%$ against Escherichia coli and for meropenem that shown a minor sensitivity reduction by $8.6 \%$ to Klebsiella species. Previous studies from Saudi found high susceptibility of Pseudomonas to carbapenems [7, 41]; however, in other local studies, the susceptibility of Pseudomonas to meropenem declined over a five-year period [29] and nonsusceptibility of Acinetobacter and Pseudomonas aeruginosa to carbapenems was very high $(68.3 \%$ and $76 \%)[6,41]$. Furthermore, there were relatively stable susceptibility patterns to all tested antimicrobials, except for cefotaxime which shown a susceptibility reduction by $41.4 \%$, among Escherichia coli and Klebsiella species; in opposite to the finding of a local study in Dhahran that shown a reduction trend in the susceptibility of antibiotics to Escherichia coli and Klebsiella species [7]. Moreover, we observed an increase in the susceptibility of Acinetobacter and Enterobacter and Citrobacter species to all studied antimicrobials except for colistin that had a slight sensitivity reduction by 4.3\% against Acinetobacter species. This can be considered as a part of the success of the combating strategies 
implemented since January 2014 at the medical settings to reduce further emergence and spread of AMR, lower the percentage of HAIs and MDR organisms, and save on needless healthcare expenses [1].

Significant differences in antibiogram findings between different healthcare facilities and regions may suggest differences in populations of the served patients, patterns of antimicrobial use, or deficiencies in hospital infection control and hygiene practices that could be further explored.

\section{Limitations}

This study had a few limitations. Firstly, the retrospective design and the risk of misclassification and selection bias. For instance, even though the laboratories follow the highest standards, there may be a possibility that some isolates had some contaminants. Furthermore, since all three hospitals in this study are tertiary care hospitals, they receive more complicated cases that may be caused by resistant pathogens which may not indicate the antibiotic susceptibility trend and microbiology of the general population. Nevertheless, our study's findings will add to local and global data on antimicrobial susceptibility, especially with highly threatening infections.

\section{Conclusion}

Systematic collection and analysis of routine clinical laboratory data is important in assessing the antimicrobial resistance burden. Nationwide surveillance is urgently needed to provide policy makers, antimicrobial stewardship committees, infection preventionists, microbiologists, and epidemiologists with essential information to guide proper action plans. The observed increase in susceptibility of gram-positive and gram-negative bacteria to studied antimicrobials is important; however, reduced sensitivity of MRSA, CoNS and Enterococcus species to gentamicin; and increased resistance of MRSA to linezolid and vancomycin is a serious threat and calls for effective antimicrobial stewardship programs.

\section{Abbreviations \\ AMS: Antimicrobial Stewardship; HAls: Healthcare Associated Infections; MRSA: Methicillin-resistant Staphylococcus aureus; VRE: Vancomycin-resistant Entero- cocci; CoNS: Coagulase-negative Staphylococci; CLABSI: Central line associated bloodstream infection; CAUTI: Catheter-associated urinary tract infection; SSI: Surgical site infection; VAE: Ventilator-associated events; HMG: Habib Medical Group; AMR: Antimicrobial resistance; MANOVA: Multivariate analysis of variance; ICUs: Intensive care units; CLSI: Clinical and Laboratory Standards Institute; EUCAST: European Committee on Antimicrobial Susceptibility Test- ing; ATCC: American Type Culture Collection.}

\section{Acknowledgements}

The authors would like to thank the Microbiology staff at Altakhassusi, Arryan and Qassim HMG Hospitals in Riyadh and Qassim, Saudi Arabia, for identifying all the isolates and for their assistance with data collection. We would also like to thank the reviewers for very helpful and valuable comments and suggestions for improving the paper.

\section{Authors' contributions}

Conceptualization, SA, AA and ZA; methodology, SA, AA and AAO; formal analysis, SA, ZA, and AAR; data curation, SA; writing - original draft preparation, SA, AA and AAR; writing - review and editing, SA, AA, ZA, AAR, AJA, AMA, $I B, I A, M T, N A, M A, A H A, F A, H A$ and $A A O$; supervision, SA, AA and AAO; project administration, SA and AA. All authors read and approved the final manuscript.

\section{Funding}

This research received no external funding.

Availability of data and materials

Data are available upon request, please contact author for data requests.

\section{Declarations}

Ethics approval and consent to participate

Ethical approval of the current study was obtained from Dr. Sulaiman Habib Institutional Review Board (RC20.10.95-2).

\section{Consent for publication}

All authors agreed to this publication.

\section{Competing of interest}

The authors have no conflicts of interest to declare.

\section{Author details}

${ }^{1}$ Administration of Pharmaceutical Care, Alahsa Health Cluster, Ministry of Health, Rashdiah Street, P. O. Box 12944, Alahsa 31982, Saudi Arabia.

${ }^{2}$ Research Center, Almoosa Specialist Hospital, Alahsa, Saudi Arabia. ${ }^{3}$ School of Nursing, Wollongong University, Wollongong, Australia. ${ }^{4}$ Department of Pediatrics, College of Medicine, King Faisal University, Alahsa, Saudi Arabia. ${ }^{5}$ College of Medicine, Al-Imam Mohammed Ibn Saud Islamic University, Riyadh, Saudi Arabia. ${ }^{6}$ Department of Pharmacology, College of Medicine, University of Jeddah, Jeddah, Saudi Arabia. ${ }^{7}$ Naif Alhmeed, Administration of Supply and Shared Services, Ministry of Health, Riyadh, Saudi Arabia. ${ }^{8}$ Pharmacy Department, King Faisal General Hospital, Alahsa, Saudi Arabia. ${ }^{9}$ Pharmacy Department, Prince Saud Bin Jalawi Hospital, Alahsa, Saudi Arabia. ${ }^{10}$ Virology Department, Regional Laboratory and Blood Bank, Riyadh, Saudi Arabia. ${ }^{11}$ Department of Histopathology, King Saud Medical City, Riyadh, Saudi Arabia. ${ }^{12}$ Molecular Diagnostics Laboratory, Johns Hopkins Aramco Healthcare, Dhahran, Saudi Arabia. ${ }^{13}$ College of Medicine, Alfaisal University, Riyadh, Saudi Arabia. ${ }^{14}$ Research Center, Dr. Sulaiman Al Habib Medical Group, Riyadh, Saudi Arabia.

Received: 23 November 2020 Accepted: 2 June 2021

Published online: 12 June 2021

\section{References}

1. Al-Omari A, Al Mutair A, Alhumaid S, Salih S, Alanazi A, Albarsan H, Abourayan M, AI SM. The impact of antimicrobial stewardship program implementation at four tertiary private hospitals: results of a five-years pre-post analysis. Antimicrob Resist Infect Control. 2020;9(1):1-9.

2. Cassini A, Högberg LD, Plachouras D, Quattrocchi A, Hoxha A, Simonsen GS, Colomb-Cotinat M, Kretzschmar ME, Devleesschauwer B, Cecchini M. Attributable deaths and disability-adjusted life-years caused by infections with antibiotic-resistant bacteria in the EU and the European Economic Area in 2015: a population-level modelling analysis. Lancet Infect Dis. 2019;19(1):56-66.

3. O'neill J. Review on antimicrobial resistance. Tackling drug-resistant infection globally: final report and recommendations. UK Government, Wellcome Trust. 2016.

4. Alrasheedy AA, Alsalloum MA, Almuqbil FA, Almuzaini MA, Aba Alkhayl BS, Albishri AS, Alharbi FF, Alharbi SR, Alodhayb AK, Alfadl AA. The impact of law enforcement on dispensing antibiotics without prescription: a 
multi-methods study from Saudi Arabia. Expert Rev Anti Infect Ther. 2020;18(1):87-97.

5. Al Mutair A, Alhumaid S, Al Alawi Z, Zaidi ARZ, Alzahrani AJ, Al-Tawfiq JA, Al-Shammari H, Rabaan AA, Khojah O, Al-Omari A. Five-year resistance trends in pathogens causing healthcare-associated infections at a multi-hospital healthcare system in Saudi Arabia, 2015-2019. J Global Antimicrob Resist. 2021;25:142-50.

6. Balkhy HH, El-Saed A, Alshamrani MM, Alsaedi A, Al Nasser W, El Gammal A, Aljohany SM, Almunif S, Arabi Y, Alqahtani S. Ten-year resistance trends in pathogens causing healthcare-associated infections; reflection of infection control interventions at a multi-hospital healthcare system in Saudi Arabia, 2007-2016. Antimicrob Resist Infect Control. 2020;9(1):21.

7. Al-Tawfiq JA, Rabaan AA, Saunar JV, Bazzi AM. Antimicrobial resistance of gram-negative bacteria: a six-year longitudinal study in a hospital in Saudi Arabia. J Infect Public Health. 2020;13:737-45.

8. Almangour TA, Alenazi B, Ghonem L, Alhifany AA, Aldakheel BA, Alruwaili A. Inhaled colistin for the treatment of nosocomial pneumonia due to multidrug-resistant gram-negative bacteria: a real-life experience in tertiary care hospitals in Saudi Arabia. Saudi Pharmaceut J. 2020;28(8):1009-13.

9. Batool S, Almaghaslah D, Alqahtani A, Almanasef M, Alasmari M, Vasudevan R, Attique S, Riaz F. Aetiology and antimicrobial susceptibility pattern of bacterial isolates in community acquired pneumonia patients at Asir region, Saudi Arabia. Int J Clin Pract. 2020;75:e13667.

10. Bandy A, Almaeen AH. Pathogenic spectrum of blood stream infections and resistance pattern in gram-negative bacteria from Aljouf region of Saudi Arabia. PLoS ONE. 2020;15(6):e0233704.

11. Institute CaLS. M100|Performance standards for antimicrobial susceptibility testing. 30th Edn; 2020.

12. Ibrahim ME, Abbas M, Al-Shahrai AM, Elamin BK. Phenotypic characterization and antibiotic resistance patterns of extended-spectrum $\beta$-Lactamase-and AmpC $\beta$-lactamase-producing gram-negative bacteria in a referral hospital, Saudi Arabia. Canadian J Infect Dis Med Microbiol. 2019;2019:1-9.

13. El-Badawy MF, Abdelwahab SF, Alghamdi SA, Shohayeb MM. Characterization of phenotypic and genotypic traits of carbapenem-resistant Acinetobacter baumannii clinical isolates recovered from a tertiary care hospital in Taif, Saudi Arabia. Infect Drug Resist. 2019;12:3113.

14. Farman M, Yasir M, Al-Hindi RR, Farraj SA, Jiman-Fatani AA, Alawi M, Azhar El. Genomic analysis of multidrug-resistant clinical Enterococcus faecalis isolates for antimicrobial resistance genes and virulence factors from the western region of Saudi Arabia. Antimicrob Resist Infect Control. 2019;8(1):55.

15. Aldrazi FA, Rabaan AA, Alsuliman SA, Aldrazi HA, Alabdalslam MJ, Alsadiq SA, Alhani HM, Bueid AS. ESBL expression and antibiotic resistance patterns in a hospital in Saudi Arabia: do healthcare staff have the whole picture? J Infect Public Health. 2020;13(5):759-66.

16. Albarrag A, Shami A, Almutairi A, Alsudairi S, Aldakeel S, Al-Amodi A. Prevalence and molecular genetics of methicillin-resistant Staphylococcus aureus colonization in nursing homes in Saudi Arabia. Canadian J Infect Dis Med Microbiol. 2020;2020:1-6.

17. Alqasim A, Jaffal AA, Almutairi N, Arshad M, Alyousef AA. Isolation, phenotypic and genotypic characterization of Escherichia coli from the bloodstream samples in Riyadh, Saudi Arabia. J King Saud Univ Sci. 2020;32(2):1464-9.

18. Al Bshabshe A, Al-Hakami A, Alshehri B, Al-Shahrani KA, Alshehri AA, Al Shahrani MB, Assiry I, Joseph MR, Alkahtani A, Hamid ME. Rising Klebsiella pneumoniae infections and its expanding drug resistance in the intensive care unit of a tertiary Healthcare Hospital, Saudi Arabia. Cureus. 2020;12(8).

19. Alsaleh NA, Al-Omar HA, Mayet AY, Mullen AB. Evaluating the appropriateness of carbapenem and piperacillin-tazobactam prescribing in a tertiary care hospital in Saudi Arabia. Saudi Pharmaceut J. 2020;28:1492-8.

20. Alnimr AM, Alamri AM. Antimicrobial activity of cephalosporin-beta-lactamase inhibitor combinations against drug-susceptible and drug-resistant Pseudomonas aeruginosa strains. J Taibah Univ Med Sci. 2020;15(3):203.

21. Alghoribi MF, Doumith M, Upton M, Al Johani SM, Alzayer M, Woodford $\mathrm{N}$, Ellington MJ, Balkhy HH. Complete Genome Sequence of a ColistinResistant Uropathogenic Escherichia coli Sequence Type 131 fimH22 Strain Harboring mcr-1 on an IncHI2 Plasmid, Isolated in Riyadh, Saudi Arabia. Microbiology resource announcements. 2019;8(18).
22. Ishaqui AA, Khan AH, Sulaiman SAS, Alsultan MT, Khan I, Naqvi AA. Assessment of efficacy of Oseltamivir-Azithromycin combination therapy in prevention of Influenza-A (H1N1) pdm09 infection complications and rapidity of symptoms relief. Expert Rev Respir Med. 2020;14(5):533-41.

23. Aljihani SA, Alehaideb Z, Alarfaj RE, Alghoribi MF, Akiel MA, Alenazi TH, Al-Fahad AJ, Al Tamimi SM, Albakr TM, Alshehri A. Enhancing azithromycin antibacterial activity by encapsulation in liposomes/liposomal- $\mathrm{N}$-acetylcysteine formulations against resistant clinical strains of Escherichia coli. Saudi J Biol Sci. 2020;27(11):3065-71.

24. Eed EM, Hawash YA, Khalifa AS, Alsharif KF, Alghamdi SA, Saber T, Ismail KA, Shehab-Eldeen SA. Molecular diagnosis of Helicobacter pylori antibiotic resistance in the Taif region, Saudi Arabia. Microbiol Immunol. 2019;63(6):199-205.

25. Al-Zahrani IA, Al-Ahmadi BM. Dissemination of VIM-producing Pseudomonas aeruginosa associated with high-risk clone ST654 in a tertiary and quaternary hospital in Makkah, Saudi Arabia. J Chemother. 2020;33:1-9.

26. El-Badawy MF, Alrobaian MM, Shohayeb MM, Abdelwahab SF. Investigation of six plasmid-mediated quinolone resistance genes among clinical isolates of pseudomonas: a genotypic study in Saudi Arabia. Infect Drug Resist. 2019;12:915.

27. Balkhy HH, El-Saed A, Alshamrani MM, Alsaedi A, Al Nasser W, El Gammal A, Aljohany SM, Arabi Y, Alqahtani S, Bonnie HB. High Burden of resistant gram negative pathogens causing device-associated healthcare infections in a tertiary care setting in Saudi Arabia, 2008-2016. J Global Antimicrob Resist. 2020;23:26-32.

28. El-Saed A, Balkhy HH, Alshamrani MM, Aljohani S, Alsaedi A, Al Nasser W, El Gammal A, Almohrij SA, Alyousef Z, Almunif S. High contribution and impact of resistant gram negative pathogens causing surgical site infections at a multi-hospital healthcare system in Saudi Arabia, 2007-2016. BMC Infect Dis. 2020;20:1-9.

29. Farah SM, Alshehri MA, Alfawaz TS, Alasmeri FA, Alageel AA, Alshahrani DA. Trends in antimicrobial susceptibility patterns in King Fahad Medical City, Riyadh, Saudi Arabia. Saudi Med J. 2019;40(3):252.

30. Haseeb A, Faidah HS, Bakhsh AR, AlMalki WH, Elrggal ME, Saleem F, ur Rahman S, Khan TM, Hassali MA. Antimicrobial resistance among pilgrims: a retrospective study from two hospitals in Makkah, Saudi Arabia. Int J Infect Dis. 2016;47:92-4.

31. Asghar AH, Faidah HS. Frequency and antimicrobial susceptibility of gram-negative bacteria isolated from 2 hospitals in Makkah, Saudi Arabia. Saudi Med J. 2009;30(8):1017-23.

32. Al-Tawfiq JA, Anani AA. Antimicrobial susceptibility pattern of bacterial pathogens causing urinary tract infections in a Saudi Arabian hospital. Chemotherapy. 2009;55(2):127-31.

33. Ibrahim ME. High antimicrobial resistant rates among gram-negative pathogens in intensive care units: a retrospective study at a tertiary care hospital in Southwest Saudi Arabia. Saudi Med J. 2018;39(10):1035.

34. Almalki MA, Varghese R. Prevalence of catheter associated biofilm producing bacteria and their antibiotic sensitivity pattern. J King Saud Univ Sci. 2020;32(2):1427-33.

35. Al Johani SM, Akhter J, Balkhy H, El-Saed A, Younan M, Memish Z. Prevalence of antimicrobial resistance among gram-negative isolates in an adult intensive care unit at a tertiary care center in Saudi Arabia. Ann Saudi Med. 2010;30(5):364-9.

36. Memish ZA, Shibl AM, Kambal AM, Ohaly YA, Ishaq A, Livermore DM. Antimicrobial resistance among non-fermenting gram-negative bacteria in Saudi Arabia. J Antimicrob Chemother. 2012;67(7):1701-5.

37. Paquette F, Bernier-Jean A, Brunette V, Ammann H, Lavergne V, Pichette $V$, Troyanov S, Bouchard J. Acute kidney injury and renal recovery with the use of aminoglycosides: a large retrospective study. Nephron. 2015;131(3):153-60.

38. Beibei L, Yun C, Mengli C, Nan B, Xuhong Y, Rui W. Linezolid versus vancomycin for the treatment of gram-positive bacterial infections: meta-analysis of randomised controlled trials. Int J Antimicrob Agents. 2010;35(1):3-12.

39. Balkhy HH, El-Saed A, El-Metwally A, Arabi YM, Aljohany SM, Al Zaibag $\mathrm{M}$, Baharoon S, Alothman AF. Antimicrobial consumption in five adult intensive care units: a 33-month surveillance study. Antimicrob Resist Infect Control. 2018;7(1):156. 
40. Balkhy H, Al Othman A, Bonnie IB, Arabi Y, El-Saed A. Consumption of carbapenems in different intensive care units in a Saudi tertiary care hospital. Antimicrob Resist Infect Control. 2015;4(1):1-.

41. Abdallah M, Badawi M, Amirah MF, Rasheed A, Mady AF, Alodat M, Alharthy A. Impact of carbapenem restriction on the antimicrobial susceptibility pattern of Pseudomonas aeruginosa isolates in the ICU. J Antimicrob Chemother. 2017;72(11):3187-90.

42. Al Bshabshe A, Joseph MR, Awad El-Gied AA, Fadul AN, Chandramoorthy $\mathrm{HC}$, Hamid ME. Clinical relevance and antimicrobial profiling of methicillin-resistant Staphylococcus aureus (MRSA) on routine antibiotics and ethanol extract of Mango Kernel (Mangifera indica L.). BioMed Res Int. 2020:2020:1-8

\section{Publisher's Note}

Springer Nature remains neutral with regard to jurisdictional claims in published maps and institutional affiliations.
Ready to submit your research? Choose BMC and benefit from:

- fast, convenient online submission

- thorough peer review by experienced researchers in your field

- rapid publication on acceptance

- support for research data, including large and complex data types

- gold Open Access which fosters wider collaboration and increased citations

- maximum visibility for your research: over 100M website views per year

At BMC, research is always in progress.

Learn more biomedcentral.com/submissions 